\title{
A Fully Immersive Virtual Reality Method for Upper Limb Rehabilitation in Spinal Cord Injury
}

\author{
Da Young Lim, MD', Dong Min Hwang, $\mathrm{MD}^{1}$, Kang Hee Cho, MD, PhD ${ }^{1,2}$, \\ Chang Won Moon, $\mathrm{MD}^{1}$, So Young Ahn, $\mathrm{MD}, \mathrm{PhD}^{1}$ \\ ${ }^{1}$ Department of Rehabilitation Medicine, Chungnam National University College of Medicine, Daejeon; \\ ${ }^{2}$ Biomedical Institute, Chungnam National University, Daejeon, Korea
}

\begin{abstract}
Objective To determine whether a fully immersive virtual reality (VR) intervention combined with conventional rehabilitation (CR) can improve upper limb function more than CR alone in patients with spinal cord injury (SCI), we conducted a prospective, randomized, controlled clinical trial.

Methods Participants were randomly assigned to either the control group (CG; $n=10$ ) or experimental group (EG; $\mathrm{n}=10$ ). The participants in the CG received 60 minutes of conventional therapy per day, 4 days per week for 4 weeks, whereas those in the EG received 30 minutes of VR training and 30 minutes of conventional therapy per day, 4 days per week for 4 weeks. The clinical outcome measures included Medical Research Council grade, the American Spinal Injury Association upper extremity motor score (ASIA-UEMS), and scores in the Hand Strength Test, Box and Block Test, Nine-Hole Peg Test, Action Research Arm Test, and Korean version of the Spinal Cord Independence Measure (K-SCIM). The assessments were performed at the beginning (T0) and end of the intervention (T1).

Results Grip power and K-SCIM score significantly improved in the EG after the intervention. When comparing differences between the groups, elbow extensor, wrist extensor, ASIA-UEMS, grip power, lateral pinch power, and palmar pinch power were all significantly improved.

Conclusion VR training of upper limb function after SCI can provide an acceptable adjunctive rehabilitation method without significant adverse effects.
\end{abstract}

Keywords Virtual reality, Rehabilitation, Spinal cord injuries

\section{INTRODUCTION}

Spinal cord injury (SCI) causes motor and sensory defi- cits below the damaged level, reducing patient quality of life [1]. Depending on the level of injury, SCI can lead to serious complications, including autonomic dysreflexia,

Received October 23, 2019; Revised November 19, 2019; Accepted December 6, 2019; Published online July 28, 2020

Corresponding author: So Young Ahn

Department of Rehabilitation Medicine, Chungnam National University Hospital, Chungnam National University College of Medicine, 282 Munhwaro, Jung-gu, Daejeon 35015, Korea. Tel: +82-42-338-2460, Fax: +82-42-338-2461, E-mail: asyoung@cnuh.co.kr

ORCID: Da Young Lim (http://orcid.org/0000-0002-5475-0830); Dong Min Hwang (http://orcid.org/0000-0002-3275-3651); Kang Hee Cho (http:// orcid.org/0000-0002-6592-3370); Chang Won Moon (http://orcid.org/0000-0003-4096-7470); So Young Ahn (http://orcid.org/0000-0002-7447-0617).

(c) This is an open-access article distributed under the terms of the Creative Commons Attribution Non-Commercial License (http://creativecommons.org/ licenses/by-nc/4.0) which permits unrestricted noncommercial use, distribution, and reproduction in any medium, provided the original work is properly cited. Copyright (C) 2020 by Korean Academy of Rehabilitation Medicine 
spasticity, respiratory system impairment, disturbances of the urinary and gastrointestinal systems, and sexual dysfunction.

The prevalence of SCI worldwide ranges from 10 to 100 cases per million individuals [2]. Approximately $60 \%$ of spinal cord-related injuries are cervical spinal cord injuries [3], which often involve severe losses of arm and hand functions and diminish life satisfaction [4]. As upper limb function plays a key role in independent daily activities such as self-management, respiration and sphincter management, and mobility, improving the upper extremity function is an important goal in rehabilitation [5].

The conventional rehabilitation (CR) is known to improve upper limb function [6], but providing sufficient treatment is labor intensive and expensive [7]. In addition, the traditional rehabilitation methods involving simple, repetitive movements may be tedious for the patient, making them less motivated to continue the treatment $[8,9]$. Therefore, new approaches must be introduced to overcome the shortcomings of the CR methods. One of the potential approaches is rehabilitation using virtual reality (VR) technology.

VR can be classified into non-immersive, semi-immersive, and fully immersive VR according to the user's immersion level. Non-immersive VR systems have been used for many years in rehabilitation therapy for the purpose of cognition, gait, and balance training, including upper extremity motor function. Many previous studies in stroke patients have reported that VR is useful for rehabilitation [10] and can achieve greater improvements than the conventional therapies [11].

In contrast to non-immersive VR systems, in which users experience both the real world and virtual environment, immersive VR systems integrate users into an environment in which all real-world perception is blocked, so only computer-generated images are seen. Headmounted display (HMD) devices are typically used to deliver a fully immersive VR experience. HMD devices can block the perception of the external environment to create a more realistic VR environment with a high level of immersion and visual scenes based on the user's movements. As immersion increases, the emotional impact of VR on the user increases, and the desired physiological response can be driven through the production of the VR experience [12]. VR can be used to motivate patients by offering various realistic sensory experiences and entertainment-based treatments $[10,13]$. Furthermore, VR can be used as a practical technology to encourage patients to exercise regularly and simulate physical movements involved in everyday life for the purpose of improving patients' capacity to cope with real-world situations $[10,11]$.

Although many VR systems have been used in stroke patients and have produced promising preliminary results $[8,10]$, few studies have examined their usefulness in patients with SCI. Thus, the purpose of the present study was to determine whether a fully immersive VR intervention combined with CR can improve upper limb function more than CR alone in patients with SCI.

\section{MATERIALS AND METHODS}

\section{Participants}

From March 2019 to July 2019, we prospectively enrolled 20 patients with upper limb dysfunction who were admitted at Chungnam National University Hospital in Daejeon, Korea. The inclusion criteria were as follows: (1) age between 19 and 75 years; (2) incomplete motor paralysis due to SCI at the $\mathrm{C} 4-\mathrm{C} 8$ neurological level; (3) $<12$ months since the onset of injury; (4) sufficient hand grip power to hold the controller; and (5) sufficient cognitive function to allow cooperation. The exclusion criteria were as follows: (1) inability to undertake the program due to pain; (2) severe spasticity (Modified Ashworth Scale grades 3 and 4); and (3) inability to sit alone. The patients were randomly assigned into two groups by using a random number table as follows: the experimental group (EG; $\mathrm{n}=10$ ) experienced fully immersive VR combined with $\mathrm{CR}$, and the control group (CG; $\mathrm{n}=10$ ) experienced $\mathrm{CR}$ alone. This study was approved by the Institutional Review Board of Chungnam National University Hospital (No. 2019-01-055) and was registered in the Clinical Research Information Service (Registration No. KCT0003786). Written informed consents were obtained.

\section{Study design}

A prospective, randomized, controlled clinical trial was conducted. The participants in the CG received $60 \mathrm{~min}$ utes of conventional occupational therapy (OT) per day, 4 days per week, for 4 weeks. The patients assigned to the CG received 30 minutes of OT by a therapist, including shoulder, elbow, wrist, and finger joint exercises; hand 
grasping-release tasks; upper extremity strengthening; and stretching, and 30 minutes of activities-of-dailyliving (ADL) training. By contrast, the participants in the EG received 30 minutes of VR training instead of ADL training and 30 minutes of OT per day, 4 days per week, for 4 weeks. The details are discussed in the VR training section.

\section{VR training}

The immersive VR device used in this study, HTC VIVE VR RehabWare (Tech Village Corp., Goyang, Korea), delivers realistic movements in the virtual world through precision controllers, HMD headsets, vivid and realistic

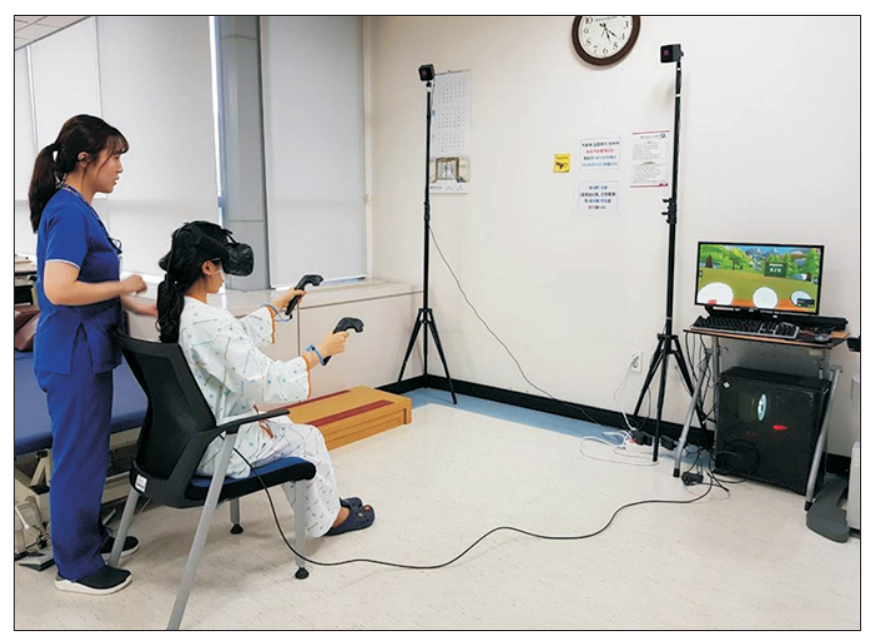

graphics, and feedback scores during the game (Fig. 1). This device tracks user movements with trackers and base stations to interact with the virtual world. Unlike stroke patients, patients with SCI typically have reduced upper extremity functions on both sides. As this device involves two controllers, it is useful for training both upper extremities. During VR training, the patient sat in the same chair with a backrest and performed six tasks (catching balls, playing xylophones, moving cherry tomatoes into a bowl, avoiding stones, throwing objects toward a target, and popping bubbles) using both hands, as shown in Fig. 2. Patients who could not remain seated were excluded, but an experienced occupational therapist always accompanied the patient as a precautionary measure to prevent harmful situations such as falling.

\section{Assessment}

The assessments were performed before the start (T0) and immediately after 4 weeks of training. Clinical outcome measures included Medical Research Council (MRC) grade, the American Spinal Injury Association upper extremity motor score (ASIA-UEMS), and scores in the Hand Strength Test (grip power, tip pinch power, lateral pinch power, and palmar pinch power), Box and Block Test (BBT), Nine-Hole Peg Test, Action Research Arm Test (ARAT), and Korean version of the Spinal Cord Independence Measure (K-SCIM).

Fig. 1. A participant wearing the virtual reality system.
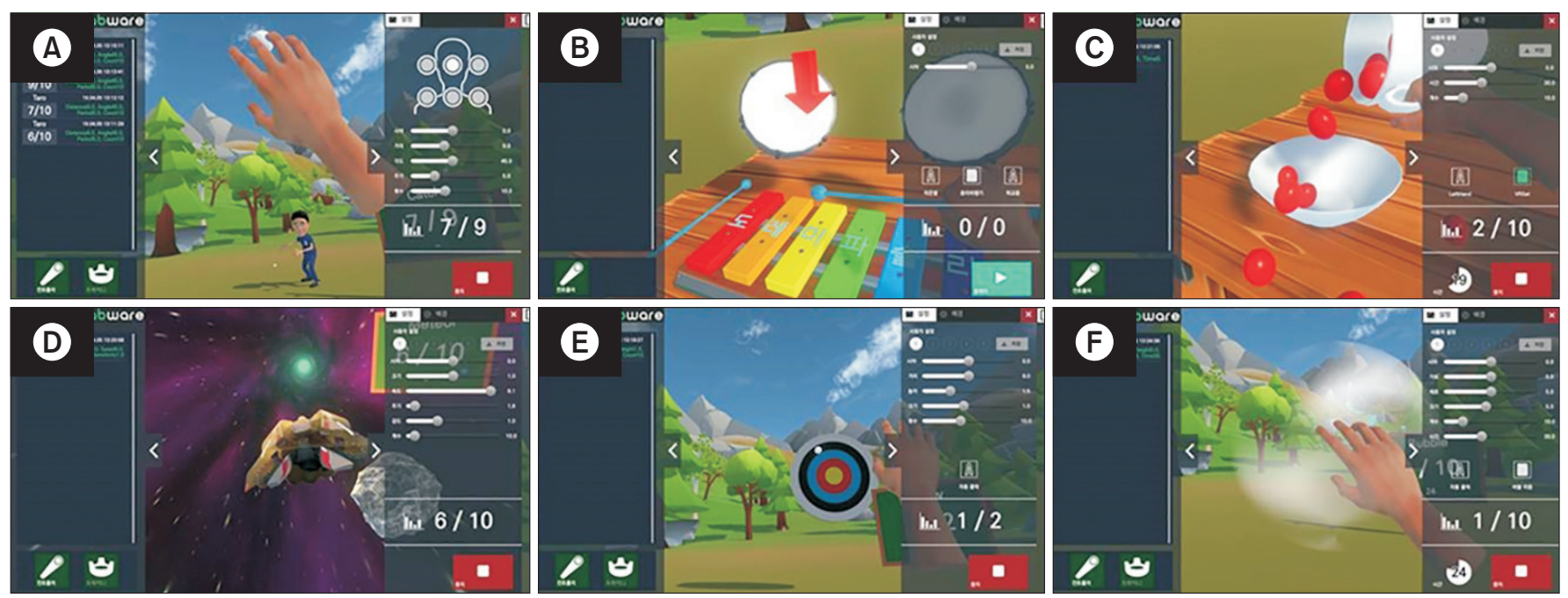

Fig. 2. Six virtual reality tasks: (A) catching balls, (B) playing xylophones, (C) moving cherry tomatoes into a bowl. (D) avoiding stones, (E) throwing objects towards a target, and (F) popping bubbles. 


\section{Arm and hand muscle strengths}

To test arm and hand muscle strengths, selected muscles (C5-T1: biceps, triceps, wrist extensor, finger flexor, and finger abductor) in both upper limbs were evaluated using the MRC grade ( $0=$ absent, $5=$ normal) in accordance with ASIA guidelines. In addition, the total scores for each limb (ASIA-UEMS) were used in the analysis. To test for grip and pinch strengths, a handheld dynamometer (BL5001 hydraulic hand dynamometer; B\&L Engineering, Santa Ana, CA, USA) and pinch gauge (Saehan SH5005 hydraulic pinch gauge; Saehan Inc., Goyang, Korea) were used. The scores in three attempts were averaged for each hand.

\section{Hand function}

For distal measurement of upper limb function, we used the BBT and Nine-Hole Peg Test, which are com- monly used to quantify hand function. The 19-item ARAT was used to measure hand function in the proximal limb, by grasping, gripping, pinching, and gross movement, rated on a 4-point scale from 0 (no movement) to 3 (movement performed normally).

\section{Independence in $A D L$}

The total K-SCIM score was measured on a scale from 0 to 100 , including the following areas of function: selfcare, respiration and sphincter management, and mobility.

\section{Statistical analysis}

All data were analyzed using the SPSS version 22.0 statistical software (IBM, Armonk, NY, USA). Continuous variables were expressed as mean \pm standard deviation, and categorical variables were expressed as a count (\%).

Table 1. Demographic and clinical characteristics of patients

\begin{tabular}{|c|c|c|c|}
\hline & $\begin{array}{c}\text { Experimental group } \\
(n=10)\end{array}$ & $\begin{array}{c}\text { Control group } \\
(n=10)\end{array}$ & p-value \\
\hline Age (yr) & $59.00 \pm 11.58$ & $61.50 \pm 12.44$ & 0.54 \\
\hline Sex, male & $6(60)$ & $8(80)$ & 0.25 \\
\hline Time since injury (day) & $27.90 \pm 8.70$ & $36.50 \pm 15.00$ & 0.32 \\
\hline Neurologic level & & & 0.86 \\
\hline $\mathrm{C} 4$ & $4(40)$ & $3(30)$ & \\
\hline C5 & $6(60)$ & $6(60)$ & \\
\hline $\mathrm{C} 6$ & $0(0)$ & $1(10)$ & \\
\hline ISNCSCI grade & & & 0.95 \\
\hline AIS-C & $0(0)$ & $1(10)$ & \\
\hline AIS-D & $10(100)$ & $9(90)$ & \\
\hline ASIA-UEMS & $19.07 \pm 1.68$ & $19.22 \pm 2.27$ & 0.93 \\
\hline \multicolumn{4}{|c|}{ Grip and pinch strength (kg) } \\
\hline Grip & $15.66 \pm 6.61$ & $22.15 \pm 7.89$ & 0.28 \\
\hline Tip pinch & $2.16 \pm 0.76$ & $4.44 \pm 1.52$ & 0.09 \\
\hline Lateral pinch & $3.29 \pm 0.98$ & $4.92 \pm 2.21$ & 0.18 \\
\hline Palmar pinch & $2.45 \pm 1.32$ & $5.05 \pm 1.93$ & 0.06 \\
\hline \multicolumn{4}{|l|}{ Hand function } \\
\hline BBT & $52.79 \pm 11.89$ & $49.72 \pm 13.49$ & 0.67 \\
\hline Nine-Hole Peg Test & $24.27 \pm 2.93$ & $33.38 \pm 23.71$ & 0.29 \\
\hline ARAT & $48.18 \pm 6.61$ & $52.27 \pm 2.31$ & 0.69 \\
\hline MMSE & $30.00 \pm 2.49$ & $28.50 \pm 2.11$ & 0.74 \\
\hline
\end{tabular}

Values are presented as mean \pm standard deviation or number (\%).

ISNCSCI, International Standard for Neurological Classification of Spinal Cord Injury; AIS, American Spinal Injury Association (ASIA) impairment scale; UEMS, upper extremity motor score; BBT, Box and Block Test; ARAT, Action Research Arm Test; MMSE, Mini-Mental State Examination. 


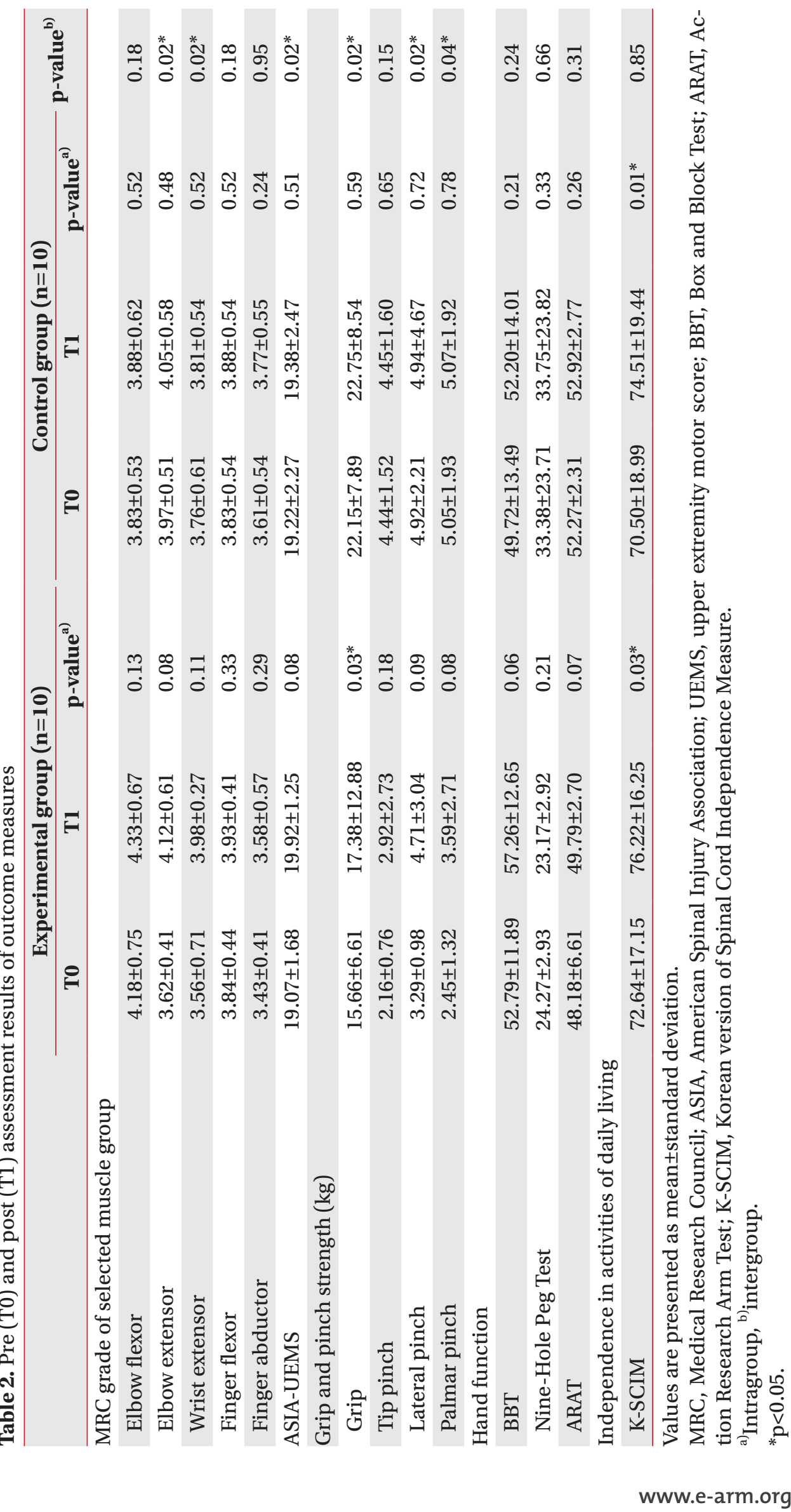


Baseline descriptive statistics were compared using the Mann-Whitney U-test for continuous data and the chisquare test for categorical data. The Wilcoxon signedrank test was used for comparison within groups both at baseline and after treatment. The Mann-Whitney U-test was used to compare differences in values between the groups before and after intervention. For all the analyses, the statistical significance was set at $\mathrm{p}<0.05$.

\section{RESULTS}

Table 1 shows the participants' baseline characteristics. The study included 10 EG participants (6 males and 4 females) and 10 CG participants (8 males and 2 females) with motor-incomplete cervical SCI. No significant differences in baseline characteristics were observed between the two groups. Both upper extremities were evaluated separately, expressing the results as mean values, but no statistical differences were found. None of the study participants experienced an adverse event due to interaction with the virtual environment (e.g., nausea, dizziness, and headache) or falls, and none of the participants dropped out of the study.

\section{Arm and hand muscle strengths}

All selective muscle strength measures were improved after intervention in both groups, but no significant differences were observed (Table 2). When comparing the differences between the groups, elbow extensor, wrist extensor, and ASIA-UEMS were significantly improved.

\section{Grip and pinch power}

Both groups exhibited improvement after intervention, but only the increases in grip power were significant $(\mathrm{p}=0.02)$ (Table 2). Significant increases in grip power, lateral pinch power, and palmar pinch power were observed in the EG participants as compared with the CG participants ( $\mathrm{p}=0.02, \mathrm{p}=0.02$, and $\mathrm{p}=0.04$, respectively).

\section{Hand function}

We found no significant differences in the hand function test scores, except for the marginally significant improvements in the BBT scores after VR treatment $(\mathrm{p}=0.06)$.

\section{Independence in ADL}

Both groups exhibited significant improvements in total
K-SCIM score, with no significant between-group differences (Table 2).

\section{DISCUSSION}

The goal of this study was to evaluate improvements of arm and hand functions and to assess the usefulness of a VR treatment program for cervical SCI as an adjunctive treatment modality for upper limb rehabilitation. The study was conducted with 16 sessions over 4 weeks, and we compared the results of VR treatment combined with CR in in the EG and those of CR alone in the CG.

This study demonstrated that VR training combined with CR resulted in functional improvement, particularly in grip power and K-SCIM score (Table 2). In addition, elbow extensor, wrist extensor, ASIA-UEMS, grip power, lateral pinch power, and palmar pinch power were all significantly improved as compared with those in the CG. Few studies have examined the VR methods in patients with SCI, and the findings of this study were inconsistent with previous studies. Dimbwadyo-Terrer et al. [14] reported that VR combined with CR showed similar results with CR alone in patients with tetraplegia. Unlike our study, that of Dimbwadyo-Terrer et al. [14] only targeted patients with motor-complete SCI (ASIAA and ASIA-B), of which ASIA-A accounted for 11 (69\%) of the 16 participants in the EG and $10(67 \%)$ of the 15 in the CG. The participants in the EG played an ADL-based VR game to improve performance in basic ADL (eating, combing hair, or washing the face) instead of using controllers but freely moved to manipulate virtual objects. Another study by Prasad et al. [15] in 2018 also reported no significant difference in hand function improvement between the two groups. The participants in the EG used a controller in this study, but the study included both subjects with motor-complete and incomplete SCI, with the sum of ASIA-A and ASIA-B accounting for $7(58 \%)$ of the 12 participants in the EG and 10 participants (70\%) in the CG, respectively. In addition, the time from SCI onset to enrollment in the present study was about a month, while those in the studies by Dimbwadyo-Terre et al. [14] and Prasad et al. [15] ranged from 4 to 5 months and from 10 to 15 months, respectively. Previous research has shown that the potential for neurological recovery was greater in incomplete SCI than in complete SCI [16], motor recovery decreases 6 months after injury $[17,18]$, early 
rehabilitation was effective, and the possibility of motor recovery was higher [19]. The discrepancy of findings between this study and the previous studies might be due to the fact that this study performed early rehabilitation for patients with motor-incomplete SCI who had been injured 1 month before or after rehabilitation.

However, previous studies in stroke patients showed consistent results. In a meta-analysis by Laver et al. [20], training with VR in stroke patients was found to improve ADL, with a moderate effect size. In another systematic review and meta-analysis, Lee et al. [21] reported that VR therapy improved muscle strength and ADL function in chronic stroke patients. Several recent studies have reported that rehabilitation using VR helped stroke patients improve their gross motor function [22]. Furthermore, several previous studies reported that VR training can be more effective and intensive than the existing treatments for improving functions in patients with damaged upper extremities due to stroke [23].

We observed functional improvements in both groups, but the EG participants in the present study may have exhibited improved hand strength because they exercised with controllers during the 30-minute intervention period, consistent with a previous study by Oh et al. [24], who reported improved hand strength after a VR intervention using a controller. Besides, the participant sees the results in the form of a score and receives positive feedback. Participants can develop advanced strategies for earning scores while viewing feedback scores during VR therapy [25]. This not only interests subjects but also triggers motor learning process.

Although improvements in upper limb function have been observed after VR treatment with CR in stroke patients, the evidence that applies to tetraplegia is still limited. Therefore, we believe that the present trial provides new insights into the role of VR applications in improving upper extremity function.

However, the present study involved several limitations that should be considered. First, the study sample was comprised of patients with tetraplegia caused by incomplete SCI only. Many previous studies examined patients with different prognoses in incomplete and complete injuries [26-28]. Therefore, patients with complete SCI must be included in future studies to determine the effects of damage due to SCI on VR treatment. Second, this was a relatively short-term study consisting of 16 rehabilitation sessions over 4 weeks. Long-term studies should be undertaken to gain a deeper understanding of the underlying mechanisms, as a short intervention period could not confirm whether VR is more effective than the traditional treatment for upper extremity rehabilitation. Finally, the patient sample in the present study was relatively small. Future studies with larger samples will be needed to confirm the present findings.

Despite the limitations of the present study, our findings demonstrate that VR training of upper limb function after SCI can provide an acceptable adjunctive rehabilitation method without significant adverse effects. In the future, larger controlled studies will be necessary to further evaluate the tolerability, feasibility, and efficacy of VR training.

\section{CONFLICT OF INTEREST}

No potential conflict of interest relevant to this article was reported.

\section{ACKNOWLEDGMENTS}

This research was financially supported by the Ministry of Trade, Industry, and Energy (MOTIE), Korea, under the Regional Industry-Based Organization Support Program (No. P0001940) supervised by the Korea Institute for Advancement of Technology.

\section{AUTHOR CONTRIBUTION}

Conceptualization: Lim DY, Ahn SY. Methodology: Lim DY, Ahn SY, Cho KH. Formal analysis: Hwang DM, Moon CW. Funding acquisition: Ahn SY. Project administration: Ahn SY. Visualization: Hwang DM, Moon CW. Writing original draft: Lim DY. Writing - review and editing: Ahn SY. Approval of final manuscript: all authors.

\section{REFERENCES}

1. Almeida VM, Paiva AE, Sena IF, Mintz A, Magno LA, Birbrair A. Pericytes make spinal cord breathless after injury. Neuroscientist 2018;24:440-7.

2. Wyndaele M, Wyndaele JJ. Incidence, prevalence and epidemiology of spinal cord injury: what learns a worldwide literature survey? Spinal Cord 2006;44:523-9. 
3. Jain NB, Ayers GD, Peterson EN, Harris MB, Morse L, O'Connor KC, et al. Traumatic spinal cord injury in the United States, 1993-2012. JAMA 2015;313:2236-43.

4. Snoek GJ, IJzerman MJ, Hermens HJ, Maxwell D, Biering-Sorensen F. Survey of the needs of patients with spinal cord injury: impact and priority for improvement in hand function in tetraplegics. Spinal Cord 2004;42:526-32.

5. Anderson KD. Targeting recovery: priorities of the spinal cord-injured population. J Neurotrauma 2004;21: 1371-83.

6. Saposnik G, Cohen LG, Mamdani M, Pooyania S, Ploughman M, Cheung D, et al. Efficacy and safety of non-immersive virtual reality exercising in stroke rehabilitation (EVREST): a randomised, multicentre, single-blind, controlled trial. Lancet Neurol 2016;15: 1019-27.

7. Kwon JS, Park MJ, Yoon IJ, Park SH. Effects of virtual reality on upper extremity function and activities of daily living performance in acute stroke: a doubleblind randomized clinical trial. NeuroRehabilitation 2012;31:379-85.

8. Lohse KR, Hilderman CG, Cheung KL, Tatla S, Van der Loos HF. Virtual reality therapy for adults post-stroke: a systematic review and meta-analysis exploring virtual environments and commercial games in therapy. PLoS One 2014;9:e93318.

9. Sin $H$, Lee $G$. Additional virtual reality training using Xbox Kinect in stroke survivors with hemiplegia. Am J Phys Med Rehabil 2013;92:871-80.

10. Saposnik G, Levin M; Outcome Research Canada (SORCan) Working Group. Virtual reality in stroke rehabilitation: a meta-analysis and implications for clinicians. Stroke 2011;42:1380-6.

11. Corbetta D, Imeri F, Gatti R. Rehabilitation that incorporates virtual reality is more effective than standard rehabilitation for improving walking speed, balance and mobility after stroke: a systematic review. J Physiother 2015;61:117-24.

12. AMP New Ventures. Virtual reality (VR) continuum [Internet]. Sydney, Australia: AMP New Ventures; 2016 [cited 2020 Jul 14]. Available from: https://www. slideshare.net/ampnewventures/virtual-reality-vrcontinuum-amp-new-ventures.

13. Moreira MC, de Amorim Lima AM, Ferraz KM, Benedetti Rodrigues MA. Use of virtual reality in gait recov- ery among post stroke patients: a systematic literature review. Disabil Rehabil Assist Technol 2013;8:357-62.

14. Dimbwadyo-Terrer I, Gil-Agudo A, Segura-Fragoso A, de los Reyes-Guzman A, Trincado-Alonso F, Piazza S, et al. Effectiveness of the virtual reality system Toyra on upper limb function in people with tetraplegia: a pilot randomized clinical trial. Biomed Res Int 2016; 2016:6397828.

15. Prasad S, Aikat R, Labani S, Khanna N. Efficacy of virtual reality in upper limb rehabilitation in patients with spinal cord injury: a pilot randomized controlled trial. Asian Spine J 2018;12:927-34.

16. Poynton AR, O'Farrell DA, Shannon F, Murray P, McManus F, Walsh MG. An evaluation of the factors affecting neurological recovery following spinal cord injury. Injury 1997;28:545-8.

17. Waters RL, Adkins RH, Yakura JS, Sie I. Motor and sensory recovery following complete tetraplegia. Arch Phys Med Rehabil 1993;74:242-7.

18. Waters RL, Adkins RH, Yakura JS, Sie I. Motor and sensory recovery following incomplete tetraplegia. Arch Phys Med Rehabil 1994;75:306-11.

19. Sumida M, Fujimoto M, Tokuhiro A, Tominaga T, Magara A, Uchida R. Early rehabilitation effect for traumatic spinal cord injury. Arch Phys Med Rehabil 2001;82:391-5.

20. Laver KE, Lange B, George S, Deutsch JE, Saposnik G, Crotty M. Virtual reality for stroke rehabilitation. Cochrane Database Syst Rev 2017;11:CD008349.

21. Lee HS, Park YJ, Park SW. The effects of virtual reality training on function in chronic stroke patients: a systematic review and meta-analysis. Biomed Res Int 2019;2019:7595639.

22. Faria AL, Andrade A, Soares L, I Badia SB. Benefits of virtual reality based cognitive rehabilitation through simulated activities of daily living: a randomized controlled trial with stroke patients. J Neuroeng Rehabil 2016;13:96.

23. Brunner I, Skouen JS, Hofstad H, Abmuss J, Becker F, Pallesen $\mathrm{H}$, et al. Is upper limb virtual reality training more intensive than conventional training for patients in the subacute phase after stroke? An analysis of treatment intensity and content. BMC Neurol 2016;16:219.

24. Oh YB, Kim GW, Han KS, Won YH, Park SH, Seo JH, et al. Efficacy of virtual reality combined with real instrument training for patients with stroke: a random- 
ized controlled trial. Arch Phys Med Rehabil 2019;100: 1400-8.

25. Harvey LA, Dunlop SA, Churilov L, Hsueh YS, Galea MP. Early intensive hand rehabilitation after spinal cord injury ("Hands On"): a protocol for a randomised controlled trial. Trials 2011;12:14.

26. Devivo MJ. Epidemiology of traumatic spinal cord injury: trends and future implications. Spinal Cord 2012; 50:365-72.

27. Bracken MB, Shepard MJ, Holford TR, Leo-Summers
L, Aldrich EF, Fazl M, et al. Administration of methylprednisolone for 24 or 48 hours or tirilazad mesylate for 48 hours in the treatment of acute spinal cord injury. Results of the Third National Acute Spinal Cord Injury Randomized Controlled Trial. JAMA 1997;277: 1597-604.

28. Bracken MB, Holford TR. Effects of timing of methylprednisolone or naloxone administration on recovery of segmental and long-tract neurological function in NASCIS 2. J Neurosurg 1993;79:500-7. 